\title{
INFLUÊNCIA AGUDA DO ALONGAMENTO ESTÁTICO E DO AQUECIMENTO AERÓBIO NO DESEMPENHO DA FORÇA MUSCULAR EM 10 REPETIÇÕES MÁXIMAS
}

\author{
ACUTE INFLUENCE OF STATIC STRETCHING AND AEROBIC HEATING IN THE PERFORMANCE OF \\ MUSCULAR STRENGTH IN 10 MAXIMUM REPETITIONS
}

\author{
Rafael André Araujo ${ }^{a^{*}}$, Rodrigo Alves França $\mathbf{a}^{\mathrm{k*}}$, Ruan Diego Schiest $\mathrm{l}^{* *}$ \\ *Grupo de Estudos em Promoção à Saúde - GEPS \\ ${ }^{* *}$ Universidade de Santa Cruz do Sul - UNISC
}

Data de entrada do artigo: 27/08/2013

Data de aceite do artigo: 19/03/2014

\section{RESUMO}

Introdução: Durante o treinamento de força, os praticantes realizam vários tipos de rotinas pré-treino, mas, na sua maioria, não sabem qual a fundamentaçáo desta preparaçáo, portanto este estudo tenta fornecer informaçôes que facilitem a escolha destas atividades. Objetivo: Avaliar a influência aguda do aquecimento aeróbio e do alongamento estático no desempenho da força máxima gerada em 10 repetiçóes máximas. Materiais e Métodos: Participaram deste estudo dez sujeitos do sexo masculino praticantes de musculação. Dias após a determinação da carga correspondente a 10 repetiçôes máximas no exercício leg press, os voluntários realizaram o máximo de repetiçôes possíveis com a mesma carga obtida anteriormente com a precedência de aquecimento aeróbio ou alongamento estático. Resultados: Em relação à média obtida nas repetiçôes em cada série, não foi encontrada diferença significativa entre as duas primeiras séries. Para as terceiras séries, foi encontrada diferença significativa entre os protocolos da mesma série. Observou-se diminuição da força muscular na terceira série do exercício, devido a fatores neuromusculares. Conclusão: Sugere-se que alongamentos nesse volume não sejam feitos antes de exercícios que requeiram grande produção de força.

Palavras-chave: Treinamento de força; alongamento; flexibilidade; aquecimento aeróbio.

\section{ABSTRACT}

Introduction: During strength training, practitioners perform various pre-workout routines, but mostly do not know what the reasons for this preparation are, therefore, this study attempts to provide information to facilitate the choice of these activities. Objective: Evaluate the acute influence of aerobic warm up and static stretching in the maximum force performance in 10 maximal repetitions. Materials and Methods: 10 male practitioners of bodybuilding participated in this study. A few days after the determination of 10 maximal repetitions at the Leg Press exercise, the volunteers performed the maximum repetitions with the same quantity of repetitions obtained previously with the precedence of aerobic warm up or static stretching. Results: Regarding the average obtained at repetitions of each set, it was not found statistic difference between the first two sets. At third set, it was found significant difference between the protocols from the same set. It was observed the diminution of the muscular strength at the third set due to neuromuscular factors. Conclusions: It is suggested that stretching in this repetition volume should not be done before exercises that require great strength production.

Keywords: Strength training; stretching; flexibility; aerobic warm up. 


\section{Introdução}

O treinamento de força é um método no qual a ação voluntária do músculo esquelético é exercida contra uma resistência externa, que pode ser desenvolvida por máquinas, peso livre ou, até mesmo, pelo próprio corpo de quem o pratica ${ }^{1}$. A prática de exercícios resistidos (ER) dentro de um treinamento de força teve grande ascensão na década de 1950, visando à reabilitação e ao desempenho esportivo, começando a ser também direcionada, durante os anos 1960, ao público adulto saudável ${ }^{2}$. Estudos indicam que os ER realizados por períodos prolongados podem ajudar a evitar doenças crônicas degenerativas ${ }^{1,3}$.

Visando a otimizaçáo do treinamento de força, diversas variáveis metodológicas são utilizadas ${ }^{4}$, embora seja possível encontrar na literatura posicionamentos conflitantes sobre os métodos que visam melhorar o ganho de força muscular. O aquecimento é uma técnica bastante empregada antes do treinamento, sendo seus benefícios relacionados ao aumento da temperatura muscular, proporcionando, assim, a melhora do metabolismo energético; aumento na elasticidade dos tecidos, no débito cardíaco e no fluxo sanguíneo; maior eficiência do sistema nervoso central e no recrutamento de unidades motoras neuromusculares ${ }^{5}$.

Os exercícios de alongamento devem fazer parte de um programa de promoçáo de flexibilidade, sendo bastante comum a utilização destes na forma de aquecimento prévio a exercícios físicos, visando à prevenção de lesões, em função da melhora da resposta das propriedades elásticas do tecido muscular e conjuntivo ${ }^{6}$. Em relação ao objetivo de prevenir lesôes, estudos presentes na literatura questionam a eficácia dos exercícios de alongamento ${ }^{4}$.

$\mathrm{Na}$ literatura ainda não há posicionamentos conclusivos no que diz respeito ao tipo de aquecimento a ser utilizado antes de um exercício ou de um treinamento de força ${ }^{7}$. Estudos apontam relação negativa entre ER precedidos de alongamento, no desempenho da força muscular ${ }^{6}$. Embora a relação entre alongamento e força ainda seja permeada por muitas controvérsias, a utilização de um aquecimento apropriado parece permitir uma execuçáo mais adequada do trabalho de força, visando os objetivos do praticante ${ }^{4}$. Portanto, o presente estudo tem por objetivo avaliar a influência aguda do aquecimento aeróbio e do alongamento estático no desempenho da força máxima gerada em 10 repetiçóes máximas.

\section{Materiais e métodos}

A amostra foi composta por dez indivíduos do sexo masculino, com pelo menos três meses de experiência na prática do treinamento de força. Foram coletados dados morfológicos dos sujeitos avaliados (tabela 1). Todos os participantes foram informados sobre os procedimentos utilizados e concordaram em participar de maneira voluntária do estudo, assinando um Termo de Consentimento Livre e Esclarecido, seguindo a resolução específica do Conselho Nacional de Saúde n ${ }^{\circ}$ 196/96. Foi aplicado o questionário "Par-Q", com o objetivo de selecionar melhor a amostra, excluindo do estudo todos aqueles que apresentassem uma ou mais respostas positivas.

A amostra foi submetida a testes de força máxima no aparelho Leg Press $45^{\circ}$ (Righetto Power Tech ${ }^{\circ}$ ), localizado no Laboratório de Estudo de Força (LABEF) da Universidade Católica de Brasília (UCB), respeitando sempre 48 horas de intervalo no mínimo entre os testes. Foram feitas três visitas no total por cada indivíduo da amostra: no $1^{\circ}$ dia realizou-se a coleta das medidas antropométricas e dos dados referentes ao teste de 10 repetiçóes máximas sem aquecimento prévio; no $2^{\circ}$ dia realizaram-se três séries, visando completar o máximo de repetiçóes possíveis com a carga obtida no $1^{\circ}$ dia, antecedidas de aquecimento aeróbio; e no $3^{\circ}$ dia foi utilizado o mesmo protocolo do $2^{\circ}$ dia, porém com alongamento estático antecedendo as séries.

$\mathrm{O}$ teste de 10 repetiçōes máximas orientou-se de acordo com as recomendações de Baechle e Earle ${ }^{8}$, em que se estimou a carga inicial de acordo com aquela habitualmente utilizada nas sessóes de treinamento de cada indivíduo. Foi definido um máximo de cinco tentativas, com intervalo de três a cinco minutos entre estas. $\mathrm{O}$ teste foi encerrado quando os avaliados foram impossibilitados de executar mais do que 10 repetiçóes máximas em razão de falha concêntrica voluntária, sendo adotada para o estudo a última carga validada. Visando reduzir ao máximo a margem de erro do teste de 10 repetiçóes máximas e com o intuito de torná-lo mais fidedigno, especificaçóes foram adotadas de acordo com Ribeiro et al. ${ }^{9}$.

O protocolo de alongamento foi composto por quatro exercícios realizados de forma estática, em duas séries nas quais o indivíduo, após atingir o limiar subjetivo de dor, permaneceu na posição por 25 segundos, com 20 segundos de intervalo entre as séries. Os exercícios são descritos como: $1^{\circ}$ ) adutores - indivíduo sentado, com os joelhos afastados e em flexão máxima, uma planta do pé pressionando a outra. Inclinar o tronco à frente; $2^{\circ}$ ) glúteos - indivíduo em decúbito dorsal, com o quadril e o joelho esquerdo flexionados em $90^{\circ}$, perna direita sobre o joelho esquerdo. Tentar aproximar a coxa esquerda do tórax. Após o procedimento com a perna esquerda, realizar o mesmo para a perna direita; $3^{\circ}$ ) isquiotibiais - indivíduo com um joelho em contato com o solo e o 
outro estendido à frente, o tornozelo à frente em flexão dorsal. Inclinar o tórax e tentar encostar a mão no pé; 4o) quadríceps - indivíduo em decúbito lateral, joelho em contato com o solo flexionado em $90^{\circ}$. Flexionar o outro joelho conduzindo o calcanhar em direçâo ao glúteo.

O aquecimento aeróbio foi realizado em um aparelho elíptico $\left(R e e b o k^{\circ}\right)$, no qual, após atingir a zona alvo de treinamento, os avaliados permaneceram nele durante seis minutos. Para o cálculo da zona alvo de treinamento, foi utilizada a fórmula FCT $=[(F C M-F C R)$ $\mathrm{x} \%$ INT] + FCR, em que FCT representa frequência cardíaca de trabalho, FCM representa frequência cardíaca máxima, FCR representa frequência cardíaca de repouso e INT representa a intensidade na qual será trabalhada $^{10}$, com intensidade entre 50 e $70 \%$ da frequência cardíaca máxima.

Depois de realizados os protocolos de aquecimento, foi respeitado um minuto de intervalo até o início do teste de repetiçóes máximas. Todos os avaliados foram instruídos a realizar três séries com o número máximo de repetiçóes, adotando dois minutos de intervalo entre elas e mobilizando a carga obtida no primeiro teste de 10 repetiçóes máximas. Para verificar a influência dos tipos de aquecimento sobre o número de repetiçóes máximas utilizou-se ANOVA one way para as medidas repetidas e teste $t$-student pareado, os dados foram plotados como média \pm desvio padrão. O nível de significância foi estabelecido em $\mathrm{p} \leq 0,05$. Todos os dados foram analisados no software Microcal Origin ${ }^{\circ}$, versão 8.0.

\section{Resultados}

A tabela 1 representa os dados morfológicos da amostra, como idade, massa corporal, estatura e IMC.

Tabela 1: Características da amostra e as medidas descritivas antropométricas

\begin{tabular}{ccccc}
\hline Variáveis & $\begin{array}{c}\text { Idade } \\
(\text { anos })\end{array}$ & $\begin{array}{c}\text { Massa } \\
\text { corporal }(\mathbf{k g})\end{array}$ & $\begin{array}{c}\text { Estatura } \\
(\mathbf{m})\end{array}$ & $\begin{array}{c}\text { IMC } \\
\left(\mathbf{k g} / \mathbf{m}^{2}\right)\end{array}$ \\
\hline $\begin{array}{c}\text { Média } \pm \\
\text { DP }\end{array}$ & $23,5 \pm 2,17$ & $76,1 \pm 13,06$ & $1,70 \pm 0,04$ & $\begin{array}{c}23,9 \pm \\
4,91\end{array}$ \\
\hline
\end{tabular}

Em relação à média obtida nas repetiçóes em cada série, não foi encontrada diferença significativa entre os diferentes protocolos de aquecimento realizados para as primeiras e segundas séries. Para as terceiras séries, foi encontrada maior diferença significativa entre os protocolos da mesma série. A figura 1 ilustra a média \pm desvio padrão das repetições em cada série.
Figura 1: A média \pm desvio padrão das repetições em cada série. ${ }^{*}$ ) diferença significativa de $p \leq 0,05$, em relação à terceira série.

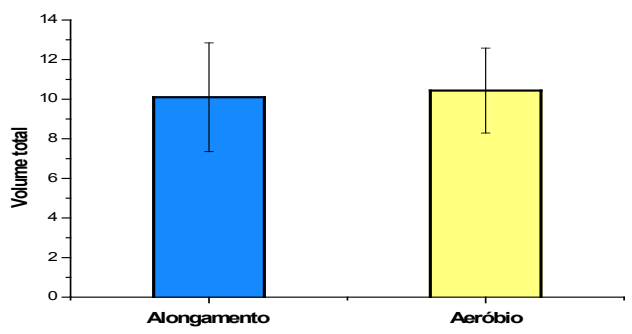

Para a média do volume total de repetições, não foi encontrada diferença significativa entre os protocolos de aquecimento. A figura 2 ilustra a média \pm desvio padrão do volume total de repetiçóes.

Figura 2: A média \pm desvio padrão do volume total de repetições

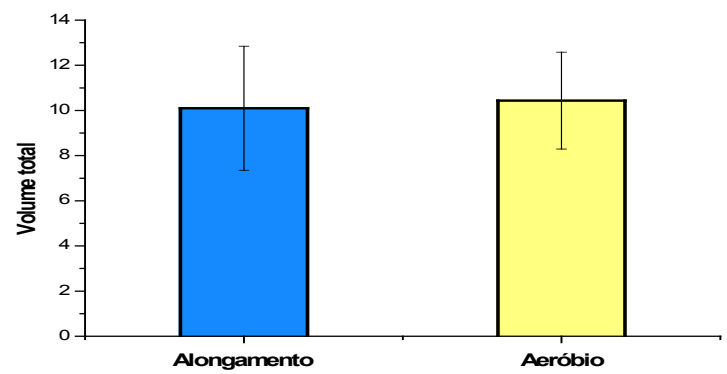

\section{Discussão}

A temática do presente estudo consistiu em comparar duas formas de aquecimento (alongamento estático e aquecimento aeróbio) realizadas previamente ao teste de 10 repetiçóes máximas com o intuito de analisar as respostas agudas dos protocolos. Não houve diferença significativa em relação ao volume total de repetições realizadas posteriormente aos protocolos de aquecimento. Em relação às séries do teste, não foi possível observar diferença significativa quando comparadas as primeiras e as segundas séries entre si.

Os resultados obtidos assemelham-se com os vistos no estudo de Fermino et al. ${ }^{11}$, no qual foram comparados o aquecimento específico e o alongamento passivo estático como forma de aquecimento, não sendo possível observar diferença significativa tanto no volume total de repetiçóes quanto na comparação (entre os protocolos) das séries do teste de carga máxima. O movimento avaliado foi a flexão de joelhos na mesa flexora e 
o protocolo de alongamento constituiu em realizar duas séries de 20 segundos, com também 20 segundos de intervalo para os músculos isquiotibiais.

No estudo realizado por Simáo et al. ${ }^{4}$, um baixo volume de alongamento por facilitação neuromuscular proprioceptiva (FNP), sendo três sustentaçóes estáticas de seis segundos na articulação escápulo-umeral, não resultou em influência significativa nas cargas máximas obtidas no exercício supino horizontal. Ainda em relação ao exercício supino horizontal, no estudo feito por Francisco, Lima e Cosenza $^{13}$ também não foi observada influência após três exercícios de alongamento, com uma série de 20 segundos, para os músculos envolvidos no gesto específico do supino.

Em 2004, Fermino et al. ${ }^{12}$ compararam a influência do aquecimento específico e o aeróbio no desempenho da força muscular em três exercícios para membros inferiores. O aquecimento aeróbio constou de 20 minutos de corrida na esteira ergométrica a $70 \%$ da frequência cardíaca de reserva. Já para o aquecimento específico, foram realizadas duas séries de 20 repetiçóes com 50\% de $1 \mathrm{RM}$. Os resultados mostram que mesmo o volume e a intensidade dos aquecimentos, sendo superiores ao do estudo realizado por Simão et al. ${ }^{7}$ não houve diferença significativa entre os protocolos propostos.

Ainda tratando-se do estudo realizado por Simáo et $a l^{7}$, os resultados mostraram que não houve diferença significativa tanto para a realizaçáo de seis exercícios de alongamento para os músculos envolvidos no exercício leg press, sendo um para cada músculo com 10 segundos de duração, quanto para 10 minutos de aquecimento aeróbio com intensidade variando entre 60 e $80 \%$ da frequência cardíaca máxima em bicicleta de ciclismo indoor. Corroborando com os resultados obtidos por Simão et al. ${ }^{7}$, até mesmo o aquecimento aeróbio com maior volume (20 minutos de corrida na esteira ergométrica), realizado no estudo de Fermino et al. ${ }^{12}$, não apontou diferença significativa entre os protocolos.

$\mathrm{Na}$ terceira série do estudo, foi possível observar uma diferença significativa para o grupo de alongamento, apontando menor número de repetiçóes no teste, quando precedido de alongamento. Resultado que corrobora com o estudo feito por Tricoli e Paulo ${ }^{14}$ que investigou a influência do alongamento estático no aparelho leg press durante o teste de uma repetição máxima. Nesse estudo, observou-se queda de rendimento apenas do grupo que realizou o teste precedido de alongamento. É importante salientar que há uma grande diferença no volume do alongamento realizado no estudo feito por Tricoli e Paulo ${ }^{14}$, em que a duração total dos exercícios de alongamento foi de 20 minutos, enquanto no presente estudo o volume total foi de aproximadamente 8 minutos.

Tal queda no rendimento devido à diminuição na produçâo de força teria relação com a possibilidade dos exercícios de alongamento possuir a capacidade de alterar as propriedades viscoelásticas da unidade músculo-tendínea, reduzindo então a tensão passiva e a rigidez do músculo ${ }^{15}$. De acordo com Fowles et al. ${ }^{16}$, exercícios de alongamento previamente ao treinamento de força poderiam ser responsáveis pelo decréscimo na ativaçáo de unidades motoras, não sendo indicados quando o objetivo for melhorar o desempenho da força muscular.

Outros estudos mostraram que alongamento estático com duração entre 15 e 30 segundos por grupamento muscular tem relação com significativa redução no recrutamento de unidades motoras ${ }^{17}$. Em um estudo usando eletromiografia, Behm et al. ${ }^{6}$ verificou redução em até $2,8 \%$ na ativação do músculo quadríceps. Tais déficits na ativação do músculo, para Kokkonen ${ }^{18}$, se explicam pelo fato de o "Órgão Tendinoso de Golgi" gerar uma inibição autogênica nos músculos alongados e em seus sinergistas, adicionando receptores de dor nos músculos e tendóes.

No presente estudo, a comparação foi feita por meio da resposta aguda, que apontou diminuiçẫo no número de repetiçóes. Se observado pelo efeito crônico, os resultados poderiam ser ainda mais expressivos, ao pensarmos que, em uma sessão de treinamento, o grupamento muscular é trabalhado com maior volume do que o realizado no estudo. Torna-se entâo importante ressaltar que se trata apenas de uma hipótese, pois a teoria dos resultados mais expressivos quando analisados do ponto de vista crônico necessita de mais estudos para elucidá-la.

\section{Conclusão}

Com base nos resultados obtidos, pode-se concluir que existe diferença estatisticamente significativa, no desempenho de força muscular em séries de 10 repetiçôes máximas no aparelho leg press em $45^{\circ}$, com a aplicação de diferentes protocolos de aquecimento.

Para o volume de alongamento utilizado, observouse diminuição da força muscular na terceira série do exercício devido a fatores neuromusculares.

Sugere-se que alongamentos nesse volume não sejam feitos antes de exercícios que requeiram grande produção de força. Sugere-se também que novos estudos com alteraçóes metodológicas e variaçốes no volume e intensidade de aquecimento sejam feitos a fim de sustentar tal afirmação.

\section{Referências}

1. Winett RA, Carpinelli RN. Potential health-related benefits of resistance training. Preventive Medicine. 2001; 33: 503-513.

2. Carpenter D, Nelson B. Low back strengthening for health, 
rehabilitation, and injury prevention. Medicine \& Science in Sports \& Exercise. 1999; 31: 18-24.

3. Fleck SJ, Kraemer WJ, RIBEIRO JL. (Trad.). Fundamentos do treinamento de força muscular. 3 ed. Porto Alegre, RS: Artmed; 2006.

4. Simão R, Giacomini MB, Dornelles TS, Marramon MG, Viveiros LE. Influência do aquecimento específico e da flexibilidade em um teste de 1 RM. Rev Brasileira de Fisiologia do Exercício. 2003; 2: 134-40.

5. Robergs RA, Roberts SO. Princípios fundamentais de fisiologia do exercício para aptidão, desempenho e saúde. São Paulo: Phorte; 2002.

6. Behm DG, Button DC, Butt JC. Factors affecting force loss with prolonged stretching. Canadian Journal Apply Physiology. 2001; B26: 261-72.

7. Simão R, Senna GW, Nassif L, Leitão N, Arruda R, Priore M, Maior AS, Polito MD. Influência dos diferentes protocolos de aquecimento na capacidade de desenvolver carga máxima no teste de 1RM. Fitness \& Performance Journal. 2004; 5: 261-5.

8. Baechle TR, Earle RW. Essentials of strength training and conditioning. Champaign: Human Kinectics, 2000.

9. Ribeiro FM, Oliveira F, Jacinto L, Santoro T, Lemos A, Simão R. Influência aguda do alongamento passivo e do aquecimento específico na capacidade de desenvolver carga máxima no teste de 10RM. Fit Perf J. 2007; 6(1): 5-9.

10. Karvonen M. et al. The effect of training on heart rate. A longitudinal study. Ann Med Exp Biol Fenn. 1957; 35: 307-315.

11. Fermino RC, Winiarski ZH, Rosa RJ, Lorenci LG, Buso
S, Simáo R. Influência do aquecimento específico e de alongamento no desempenho da força muscular em 10 repetiçóes máximas. Rev Brasileira de Cineantropometria e Desenvolvimento Humano. 2005; 13(4): 25-32.

12. Fermino RC, Kotaba C, Santos A, Zen V, Simão R, Polito M, Monteiro W. Influência de diferentes aquecimentos no desempenho da força muscular. Rev Bras Fisio Exerc. 2004; 3: 249-56.

13. Francisco RV, Lima, AJG, Cosenza PI. Efeito agudo do alongamento e de ambos no número máximo de repetiçôes realizadas com $80 \%$ de $1 \mathrm{RM}$ no supino. IN: XXVll Simpósio Internacional de Ciências do Esporte. Rev Bras Cien Mov. 2004; 12: 223.

14. Tricoli V, Paulo AC. Efeito agudo dos exercícios de alongamento sobre o desempenho de força máxima. Rev Atividade Física e Saúde. 2002; 7: 6-13.

15. Kubo K, Kanehisa H, Kawakami Y, Fukunaga T. Influence of static stretching on viscoelastic properties of human tendon structures in vivo. J Appl Phys. 2001; 90: 520-7.

16. Fowles JR, Sale DG, MacDougall JD. Reduced strength after passive stretch of the human plantarflexors. J Appl Phys. 2000; 89: 1179-88.

17. Shrier I, Gossal K. Myths and truths of stretching. Phys Sportsmed. 2000; 28: 18-25.

18. Kokkonen J, Nelson AG, Cornwell A. Acute muscle stretching inhibits maximal strength performance. Research Quarterly for Exercise and Sport. 1998; 69: 411-15. 